\title{
Strategies to prevent falls and fractures in hospitals and care homes and effect of cognitive impairment: systematic review and meta-analyses
}

\author{
David Oliver, James B Connelly, Christina R Victor, Fiona E Shaw, Anne Whitehead, Yasemin Genc, Alessandra \\ Vanoli, Finbarr C Martin, Margot A Gosney
}

\begin{abstract}
Objectives To evaluate the evidence for strategies to prevent falls or fractures in residents in care homes and hospital inpatients and to investigate the effect of dementia and cognitive impairment.

Design Systematic review and meta-analyses of studies grouped by intervention and setting (hospital or care home).

Meta-regression to investigate the effects of dementia and of study quality and design.

Data sources Medline, CINAHL, Embase, PsychInfo, Cochrane Database, Clinical Trials Register, and hand searching of references from reviews and guidelines to January 2005. Results 1207 references were identified, including 115 systematic reviews, expert reviews, or guidelines. Of the 92 full papers inspected, 43 were included. Meta-analysis for multifaceted interventions in hospital (13 studies) showed a rate ratio of 0.82 (95\% confidence interval 0.68 to 0.997$)$ for falls but no significant effect on the number of fallers or fractures. For hip protectors in care homes (11 studies) the rate ratio for hip fractures was 0.67 ( 0.46 to 0.98 ), but there was no significant effect on falls and not enough studies on fallers. For all other interventions (multifaceted interventions in care homes; removal of physical restraints in either setting; fall alarm devices in either setting; exercise in care homes; calcium/vitamin D in care homes; changes in the physical environment in either setting; medication review in hospital) meta-analysis was either unsuitable because of insufficient studies or showed no significant effect on falls, fallers, or fractures, despite strongly positive results in some individual studies. Meta-regression showed no significant association between effect size and prevalence of dementia or cognitive impairment.

Conclusion There is some evidence that multifaceted interventions in hospital reduce the number of falls and that use of hip protectors in care homes prevents hip fractures. There is insufficient evidence, however, for the effectiveness of other single interventions in hospitals or care homes or multifaceted interventions in care homes.
\end{abstract}

\section{Introduction}

Falls are common in hospitals and care homes, ${ }^{1}$ where rates vary from three to 13 falls per 1000 bed days. In 2004-5, 275000 falls were reported in hospitals in the United Kingdom ${ }^{2}-60 \%$ of all reported incidents. Cohort studies in UK care homes have shown that residents fall two to six times a year. ${ }^{3}$ About $30 \%$ of falls in hospitals and care homes result in physical injury and $3-5 \%$ in fracture. ${ }^{45}$ Up to $20 \%$ of admissions to general hospitals for hip fracture are from care homes. ${ }^{6}$ Falls may also lead to loss of function, anxiety, depression, impaired rehabilitation, increased length of hospital stay, and inability to return to previous residence, thus contributing to additional health and social care costs. ${ }^{7-9}$ Falls in institutions may result in complaints or litigation from families. ${ }^{10}{ }^{11}$ All of this leads to anxiety for staff and proprietors, who require guidance on best practice in preventing falls and injuries.

Falls often indicate underlying frailty or illness and thus require a broad approach to assessment and management. ${ }^{12}{ }^{13}$ Most evidence about successful prevention strategies, however, is derived from less frail and more clinically stable people living in their own homes. ${ }^{12} 13$ Such evidence may not translate to transient populations who are medically unstable with a high prevalence of cognitive impairment, as is typical in hospitals or care homes. We synthesised and evaluated the evidence for prevention of falls and fractures in hospitals and care homes to inform the development of guidance on best practice; to avoid implementation of ineffective or harmful strategies; and to identify gaps and controversies in the evidence from research. We also investigated the impact of cognitive impairment or dementia on the effectiveness of the identified interventions.

\section{Methods}

\section{Literature search strategy}

In accordance with QUOROM ${ }^{14}$ and Cochrane ${ }^{15}$ we searched Medline, CINAHL, Embase, PsychInfo, Cochrane Database of Systematic Reviews, and the Register of Clinical Trials for guidelines and hand searched references from 115 guidelines, systematic, or expert reviews (see www.reading.ac.uk/ihs/ bmj_falls.htm). Our core search terms were "accidental fall", "fracture", "accident prevention", "risk management", "wounds and injuries", "hospital", "hospitalisation", "residential facilities", "care homes", "institutionalisation", “dementia”, “delirium”, “cognitive disorders", "clinical trials", "restraint physical", "protective devices" (see www.reading.ac.uk/ihs/bmj_falls.htm for full search strategy). When possible, we contacted authors of included studies to ascertain their knowledge of unpublished data or ongoing trials.

References to the studies included in the systematic review (w1-w44) and three tables showing key features, quality scores, and outcome measures are on bmj.com. 


\section{Identification of articles for inclusion}

Our initial inclusion criteria were deliberately broad. We sought studies of patients in hospitals or care homes that reported the number or rate of falls or fractures or people who fell ("fallers") as a primary or secondary outcome. For inclusion, the data also had to be reported in such a way that we could calculate log rate ratios or log relative risks and their variances. We included trials with individual or cluster randomisation, case-control studies, and observational cohort studies, this variety reflecting the methodological and logistical difficulties of performing randomised controlled trials in these settings and with these populations.

\section{Abstraction of data and outcomes}

We used the quality score of Downs and Black to assess papers because it enables assessment of studies of various design. ${ }^{16}$ After initial piloting on five papers to assess inter-rater reliability, we determined that the statistical items on the score should be independently scored by a statistician (AW). We also piloted and refined a data extraction form summarising key features of trial design, study population, and interventions on these five papers. One of three pairs of peer assessors (DO/MG, JC/CV, FM/FS) scored each included study and extracted data. Each pair worked independently with a further assessor arbitrating if necessary.

\section{Classification of studies for meta-analysis}

For meta-analyses and presentation of results, we grouped studies into nine categories according to type and setting of the intervention. The prevalence of dementia in study participants was categorised by a "metric" where 0 was unknown, 1 was $<40 \%$, 2 was $40-69 \%$, and 3 was $\geq 70 \%$. If the study did not state the prevalence of dementia but the environment was a specialist setting for dementia care, then we assumed 3 .

\section{Extraction, analysis, and synthesis of quantitative outcome data}

Two statisticians (YG and AW) abstracted quantitative outcome data. The three outcomes were falls per person year, fractures/1000 person years, and percentage of people falling. Measures of the effect of the intervention relative to the control were the $\log$ rate ratio for falls and fractures and the $\log$ relative risk of falling. We calculated estimates of these effect measures and their variances for each study when data permitted. ${ }^{17} 18$ Where reports of cluster randomised trials had not reported any adjustment for clustering (as stipulated by the CONSORT statement ${ }^{19}$ ), we used the intracluster correlation coefficients of 0.1 , 0.071 , and 0.026 for falls, fractures, and fallers, respectively, provided by Dyer et al. ${ }^{\mathrm{wl}}$ We carried out a sensitivity analysis for the effect of these coefficients for meta-analyses that included five or more cluster randomised trials.

Heterogeneity between studies was quantified by the $I^{2}$ statistic. ${ }^{20}$ Fixed $^{17}$ and random ${ }^{18}$ effects meta-analyses were performed separately within each of the nine categories for each of the three principal outcome measures (falls, fallers, and fractures), provided there was a minimum of three studies. We created forest plots with studies ordered by quality score and calculated $95 \%$ confidence intervals for estimates. Because of heterogeneity between studies, we have presented only random effects models. We undertook random effects meta-regression using restricted maximum likelihood (REML) methods ${ }^{21}$ for the estimates of the regression parameters and the heterogeneity variance to assess the potential effect of dementia as an effect modifier. Dementia was considered as a categorical variable with four levels, and all studies were included in these meta-regressions. To allow for possible confounding by the type of intervention, we repeated

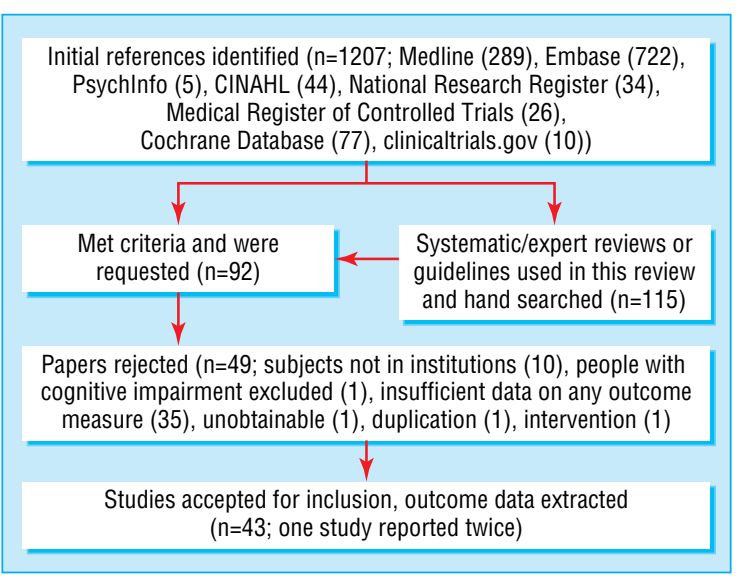

Fig 1 QUOROM flow diagram of selection of studies

meta-regressions adjusting for this factor. Finally, as we had aggregated studies of varying design and quality, we performed meta-regression to describe any association between effect size and the type or quality of studies.

\section{Results}

Figure 1 summarises the literature search, which resulted in the final inclusion of 43 studies. The full list of rejected studies along with reasons for rejection can be found on our website (www.reading.ac.uk/ihs/bmj_falls.htm).

The key features and quality scores for the 43 included studies are summarised in table A on bmj.com. Outcome measures and $95 \%$ confidence intervals are summarised in tables B and C on bmj.com. Individual elements of the Downs and Black score by paper are listed on our website (www.reading.ac.uk/ihs/ bmj_falls.htm). Sixteen studies were individually randomised controlled trials, 12 were cluster randomised, nine were prospective (historical control), two were retrospective observational cohort, two were prospective observational cohort, one was a prospective case-control study, and one was quasi-experimental with a multiple interrupted time series.

Sufficient data were available for us to calculate estimates and variances of log rate ratios for falls in 35 studies and fractures in 22 studies and for log relative risk for falling in 25 studies. Although several study populations had a high prevalence of dementia, only one study was exclusively in people with dementia or cognitive impairment ${ }^{\mathrm{w} 2}$ (though in this case, only $70 \%$ were from institutional settings). Jensen et al initially reported a cluster randomised trial from care homes, ${ }^{\mathrm{w} 3}$ but then reported subgroup analysis based on levels of cognitive impairment. ${ }^{\mathrm{w4}}$ For this review we treated the subgroups as separate datasets. Donald et al reported a $2 \times 2$ factorial design with the interventions being exercise and environmental change ${ }^{\mathrm{w5}}$ and so we included it under two separate single intervention headings.

We present our results for each of the nine categories of intervention and setting and forest plots only for three key interventions and four outcomes and only for random (not fixed) effects. Other meta-analyses are available on our website (www.reading.ac.uk/ihs/bmj_falls.htm).

\section{Multifaceted interventions}

In hospital -We included 13 studies $^{\mathrm{w6}-18}$ : three were individually randomised, two were cluster randomised trials, and eight were prospective (historical control "before and after" studies), which with one exception ${ }^{\text {w8 }}$ were of poor methodological quality. Components of interventions were varied and included risk 


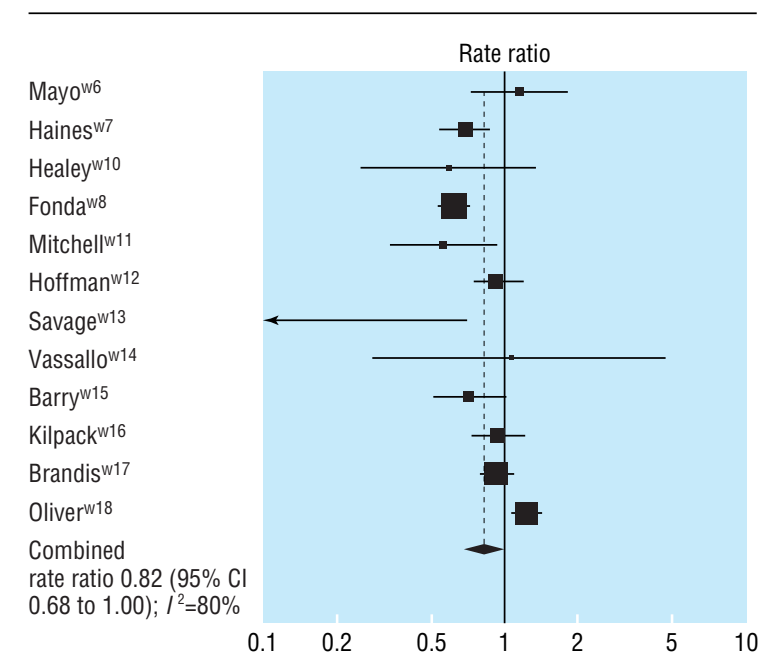

Fig 2 Meta-analysis for multifaceted interventions in hospital-falls (random effects model)

assessment, risk factor assessment, care planning, medical/ diagnostic approaches, changes in the physical environment, education programmes, medication review, hip protectors, removal of physical restraints, and exercise (see table A on bmj.com). There was evidence of heterogeneity between the studies $\left(I^{2}=80 \%\right.$ for falls, $59 \%$ for fractures, and $58 \%$ for fallers) The rate ratio was $0.82(95 \%$ confidence interval 0.68 to 0.997$)$ for falls (fig 2) and 0.59 (0.22 to 1.58) for fractures. The relative risk for fallers was 0.95 (0.71 to 1.27 ).

In care homes-We included eight studies, ${ }^{\mathrm{w1-w4}}{ }^{\text {w19-w23 }}$ one of which yielded two datasets. ${ }^{\text {w3 }}$ w4 Three were individually randomised and five were cluster randomised controlled trials. The components of the interventions were varied and included all those listed for hospital patients (see table A on bmj.com). Quality scores were generally high. There was significant heterogeneity for falls $\left(I^{2}=87 \%\right)$, although this was much less for fallers (24\%) and non-existent for fractures. The rate ratio was 0.80 (0.59 to 1.09) for falls (fig 3) and 0.91 (0.54 to 1.53) for fractures. The relative risk for fallers was 0.92 (0.82 to 1.03) (fig 4). Sensitivity analyses performed for falls and fallers, varying the intraclass correlation coefficient between 0 and 1 , had little impact on the conclusions; confidence intervals always included 1 , though the width of the random effects confidence intervals reduced with increasing correlation.

\section{Single interventions}

All the single interventions described were also components of one or more of the multifaceted interventions in care homes and hospitals.

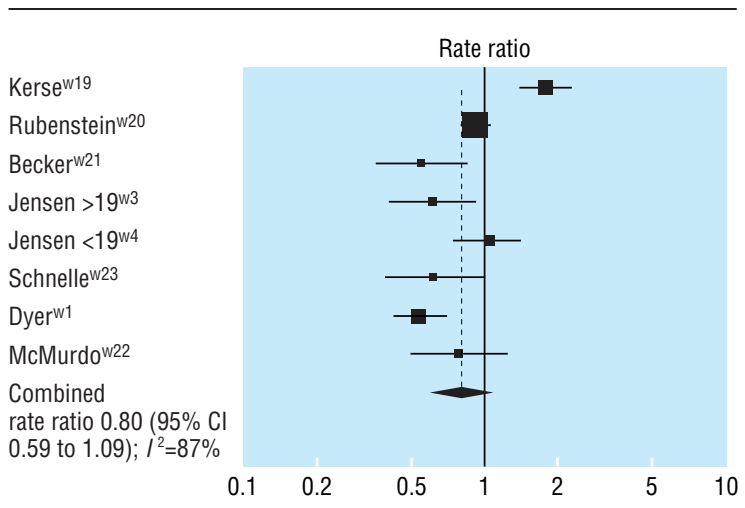

Fig 3 Meta-analysis for multifaceted interventions in care homes for falls (random effects model)

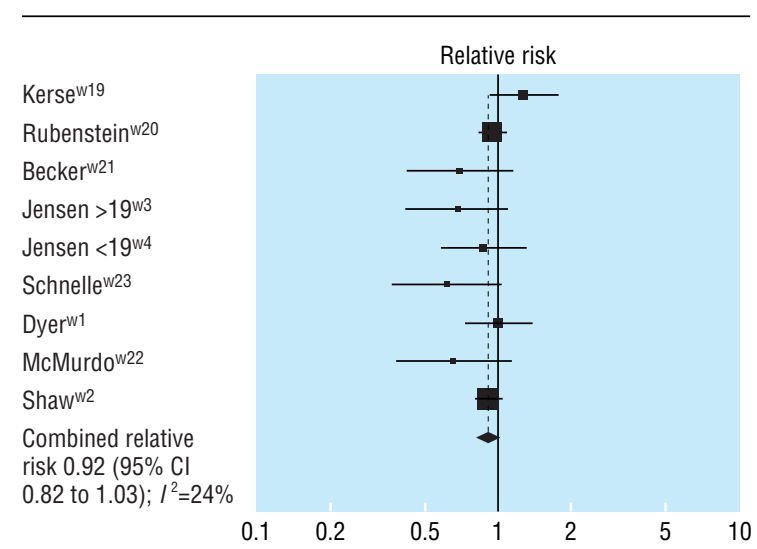

Fig 4 Meta-analysis for multifaceted interventions in care homes for fallers (random effects model). Jensen et al carried out subgroup analyses according to score on MMSE (mini-mental state examination) of $<19 v>19$

Hip protectors in care homes-We included 11 studies $^{\mathrm{w} 24-\mathrm{w} 34}$ : five were individually randomised controlled trials, five were cluster randomised controlled trials, and one was a prospective historical control study. Quality scores were generally high. There was significant heterogeneity for falls $\left(I^{2}=90 \%\right)$, but less for hip fractures (39\%). Meta-analysis was not performed for fallers as only two studies reported relevant data. The rate ratio was 0.67 (0.46 to 0.98 ) for hip fractures (fig 5) and 0.97 (0.77 to 1.22) for falls. The results for hip fractures were sensitive to the magnitude of the intraclass correlation coefficient: values of $\geq 0.05$ gave results that were not significant, whereas the value of 0.026 as reported by Dyer and colleagues ${ }^{\mathrm{wl}}$ and lower values gave significant results. The conclusions regarding hip fractures are therefore uncertain.

Removal of physical restraint in either setting-We included five studies ${ }^{\mathrm{w} 35-\mathrm{w} 39}$ : two were prospective with historical controls and three were observational cohort studies. Three were in care homes, one was in an assessment and treatment unit for older people, and one was in a hospital stroke ward. Studies were generally of moderate methodological quality. There was significant heterogeneity for falls $\left(I^{2}=99 \%\right)$ and fallers $(91 \%)$. The rate ratio was 0.59 (0.19 to 1.77 ) for falls, and the relative risk for fallers was 0.83 (0.42 to 1.66$)$. Only one study provided data on fractures.

Fall alarm devices in either setting-We included only one study: a small prospective historical control crossover study of moderate methodological quality in a care home. ${ }^{\mathrm{w} 40}$ This showed a significant effect on falls, though not on fallers or fractures. It was a small study, however, with a low quality score, making general conclusions hard to draw.

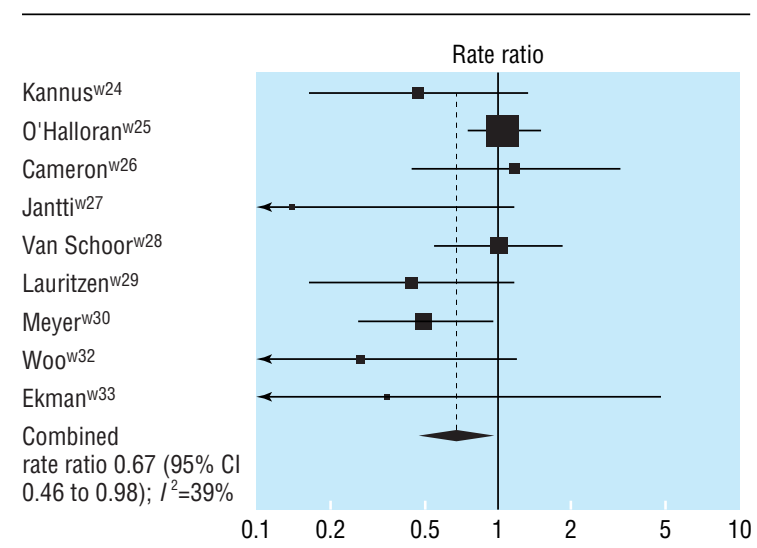

Fig 5 Meta-analysis for hip protectors as a single intervention in care homes (no hospital studies were identified)-hip fractures (random effects model) 
Exercise in either setting-We included two individually randomised controlled trials. ${ }^{\text {w5 }}{ }^{\text {w1 }}$ Neither showed any effect on falls, despite good adherence and a range of other benefits.

Changes or differences in the physical environment in either setting-We included only one study, which compared carpeting with vinyl flooring. ${ }^{\mathrm{w} 5}$ There were few participants, and there was no significant effect on the rate of falls. One review that we identified but excluded was a large observational cohort study in a UK care home that compared rates of falls and fractures in residents' rooms with concrete or wooden floors and carpeted or uncarpeted. ${ }^{22}$ They found that wooden carpeted floors were associated with the lowest rate of fractures. Also a retrospective observational cohort study of falls in a general hospital showed that falls on wooden flooring were associated with a significantly lower rate of injuries. ${ }^{23}$

Calcium and vitamin D in care homes-Only two published studies were eligible for inclusion, both individually randomised controlled trials. ${ }^{\text {w42 w43 }}$ Compared with placebo there was a significant effect on the rate of hip fracture, ${ }^{\text {w42 }}$ and compared with calcium alone there was a significant effect on the fall rate. ${ }^{\text {w43 }}$ Two key studies have been published since our review. Both were large randomised controlled trials from care homes in residents with a high prevalence of vitamin D deficiency. ${ }^{24}{ }^{25}$ One showed a significant effect on falls and fractures ${ }^{24}$ and the other found no evidence of prevention for either outcome. ${ }^{25}$

Medication review in either setting-Only one study reported review of medication as a single intervention in preventing falls. ${ }^{\text {w4 }}$ This small hospital based study reported a rate ratio for falls of 0.53 (0.30 to 0.95$)$.

\section{Meta-regression}

The results of meta-regressions to assess the effect of prevalence of dementia on effect size for each of the three reported outcome measures were not significant: the $\mathrm{P}$ values were 0.72 for the rate ratio for falls, 0.87 for the relative risk for fallers, and 0.18 for the rate ratio for fractures. After adjustment for the type of intervention, the corresponding $\mathrm{P}$ values were $0.37,0.78$, and 0.006 (see www.reading.ac.uk/ihs/bmj_falls.htm for further details). Although this suggests an association between a smaller intervention effect on fracture rate and increasing prevalence of dementia, the significant effect seemed to be caused by a difference between the "unknown" category and the others. Therefore there is no conclusive evidence that the effect size is modified by the prevalence of dementia.

For meta-regressions on study type, they were categorised into randomised controlled trials (including cluster randomised controlled trials) versus the rest. Results were not significant for falls $(P=0.19)$ or fallers $(P=0.11)$ but were for fractures $(\mathrm{P}<0.001)$. For fractures the randomised controlled trials had a ratio closer to 1 than the other studies did. The conclusions were not altered when we adjusted for type of intervention. For the assessment of study quality on the results we placed quality scores into one of three categories: $<15,15-19$, and $\geq 20$. Metaregressions on study quality were not significant for falls $(\mathrm{P}=0.41)$ or fallers $(\mathrm{P}=0.44)$ but were for fractures $(\mathrm{P}=0.004)$. For fractures, the lower the quality score the larger the effect of the intervention. After adjustment for the type of intervention, the conclusions did not change for fallers or fractures but became significant for falls $(\mathrm{P}=0.021)$. This effect with falls was difficult to interpret as the largest treatment effect was found with the middle category of study quality, with the lowest category showing a slightly negative effect.

\section{Discussion}

There have been two major approaches to interventions to reduce falls in hospital and care homes: those based on a single intervention and those that combine various interventions. In this systematic review and meta-analysis we found some evidence for modest reductions in rates of falls in hospital patients with multifaceted interventions and in rates of hip fractures with hip protectors in people in care homes. However, we found insufficient evidence for any other interventions in these settings.

Undertaking research on prevention of falls and fractures in hospitals and care homes is problematic. There are inherent logistic difficulties in performing or interpreting studies in care homes or hospitals associated with population, setting, design, and outcome measurement. Getting consent from or randomising frail, confused, unwell elderly people, who are often in the institution for only a short stay, is challenging. For outcome measures such as number of falls and fallers recorded by staff there may be recording bias.

The wide range of studies is reflected in the quality scores, which we present as a descriptive measure to guide interpretation of findings. Use of a cut-off score as an inclusion criterion would have been arbitrary and led to exclusion of results of interest in a specialty where there is still a lack of robust evidence. For answering certain types of question (for example, the effect of physical environment or restraint removal) conventional randomised controlled trial design is neither feasible nor suitable as individual randomisation within the same setting may lead to "contamination" of the control group. Cluster randomisation overcomes this, but the analysis needs adjustment for intracluster correlation.

Studies may have high internal validity for the specific setting, case mix, skill mix in staff, physical environment, and medical or nursing culture in which they were performed. Factors such as the degree of adherence to interventions or the prevalence of cognitive impairment are likely to affect the success of interventions. Finally, the content of multifaceted interventions incorporating a range of approaches differs from study to study, making the benefit of each individual component hard to attribute.

\section{Design problems}

The approach we took to the methodological challenges necessarily affects the interpretation of our findings. Categorisation by type of intervention has greater methodological validity than aggregating results from disparate interventions. We aggregated studies of different design (such as before and after and randomised controlled trials) so as not to lose useful data from studies where this design would not have been feasible. The inclusion of study setting in deciding the type was intended to enable the review's findings to be applied operationally to particular settings. Nevertheless, heterogeneity of case mix of participants or clinical practice is still likely to exist across similar settings and we could not account for this in our analyses.

With regard to the analysis of falls and fallers, differences between the studies in terms of design and quality did not seem to affect the results. With regard to fractures, however, there is some evidence that randomised controlled trials (including cluster randomised controlled trials) have smaller effects than other types of design, and that effect size increases with lower quality. When the original authors did not report intracluster correlation coefficients, we used estimates from Dyer et $\mathrm{al}^{\mathrm{w} 1}$ on the grounds that imputation of the variances was preferable to excluding the study from the meta-analysis. Sensitivity analyses undertaken for 
three of the meta-analyses heavily dependent on cluster randomised trials showed that for falls and fallers in multifaceted interventions in care homes the conclusions remained the same over a wide range of correlation coefficients. For the analysis of hip fractures and hip protectors, however, the results changed from significant to non-significant once the correlation coefficient rose above about 0.05. Finally, our insistence on including only studies for which our three standardised outcome measures could be calculated led to the exclusion of some high quality studies with potentially useful information (such as Simpson et $\mathrm{al}^{22}$ and Ray et $\left.\mathrm{al}^{26}\right)$. Standardisation of outcomes was an operational necessity for the meta-analysis. Because of the small number of studies in each intervention type, we performed meta-regressions across all interventions. Interpretation of the meta-regression to test effect size versus design and quality of studies is difficult because of the association between study design and intervention. Some interventions (such as hip protectors) were almost exclusively carried out in high quality randomised controlled trials whereas others (such as multifaceted interventions in hospital) were usually of non-randomised design. The only clear association between quality score and effect size was for fracture as an outcome.

\section{Effect of dementia}

The application of a "metric" to investigate the potential effect of dementia as an effect modifier was a pragmatic solution to a difficult problem. Only one study included solely participants with dementia, ${ }^{\mathrm{w} 2}$ and only one reported subgroup analyses to compare effects on those with or without dementia. ${ }^{\mathrm{w} 4}$ Yet there was often a high prevalence of dementia in participants, which was defined in diverse ways (for example, various mental test scores, clinical diagnosis, behavioural rating scales).

\section{Key findings}

We identified 13 studies with multifaceted approaches to preventing falls in hospital. The components of these interventions were varied, the settings and populations heterogeneous, and the study design and quality highly variable. Two high quality randomised controlled trials ${ }^{\mathrm{w} 7 \mathrm{w10}}$ and one high quality before and after study ${ }^{\text {w8 }}$ described significant effects on rates of falls, with meta-analysis showing a reduction of $18 \%$, which was just significant, though with no comparable effect on fractures or fallers. Of eight studies reporting multifaceted interventions in care homes, all were described as randomised controlled trials and were generally of good quality, though adjustments for clustering were rarely undertaken in the original analyses. While some individual trials showed a large effect size on falls (though not on fractures or fallers), ${ }^{\text {w3 }}{ }^{\text {w21 }}$ the meta-analyses for multifaceted interventions in care homes did not show any significant effects. There was insufficient power to detect a difference in fracture rates in these studies so the results are inconclusive.

We identified 11 studies on the use of hip protectors, 10 of which were randomised controlled trials. There was evidence to show an overall effect of hip protectors on rates of hip fracture. Given the number of cluster randomised trials in this meta-analysis and the sensitivity of the results to the magnitude of the intraclass correlation coefficient, which necessarily had to be imputed for most studies, we consider it unwise to draw definite conclusions about efficacy. There was no evidence for exercise as a single intervention in preventing falls or fractures, though it was a component of several successful multifaceted interventions and conferred a range of other benefits. Five studies on removal of physical restraint (of necessity, largely with observational cohort or case control designs) showed no evidence of significant effect on falls or fractures in either direction. There were not enough trials of fall alarm devices, changes in physical environment, or medication review as single interventions. Oral calcium and vitamin D at appropriate doses reduced rates of falls ${ }^{\mathrm{w} 42,}$ w43 and fractures ${ }^{\mathrm{w4} 43}$ for older people in care homes in the two included studies. A recent randomised controlled trial in care home residents in Australia ${ }^{24}$ mirrored these findings (though it was published too late for inclusion), but a subsequent UK study ${ }^{25}$ failed to replicate them, confusing the picture. Many of the single interventions described were also components of multifaceted interventions.

Our findings suggest that there is reasonable evidence that using a structured multifaceted intervention for hospital inpatients may have a modest effect on falls but not on fractures. Replication of such studies would not be a high priority, but closer investigation of specific components is required. A similar approach in care homes has yielded significant reductions in falls, fallers, and fractures in some large individual studies, but this is not substantiated by meta-analysis so the case for effectiveness is unproved. The use of high dose calcium and vitamin D has proved effective in three studies in care homes but ineffective in a more recent and larger study, suggesting that effects might be setting and population specific. For all other interventions listed, there is currently no evidence to support widespread implementation. With fractures as an outcome, for all interventions except for hip protectors there was insufficient power to detect a significant difference, so that the case remains unproved rather than disproved.

Our review illustrates an increasingly prevalent view ${ }^{27}$ that an over-reliance on the primacy of the randomised controlled trial as the main source of clinical evidence may not be suitable where interventions are complex and individual consent is hard to obtain. An approach of realistic evaluation, ${ }^{28}$ where factors such as context, case mix, adherence, quality of intervention, and process are considered, may be more suitable in answering many pragmatic questions around "real life" clinical practice. ${ }^{29} 30$

\section{Conclusions}

We have identified many gaps in the evidence that merit further investigation-for example, interventions specifically for those with cognitive impairment or dementia; the reproducibility of interventions within and between different types of service setting; cost effectiveness of interventions; and the effect of a range of single interventions, such as medication review, use of alarms, or changes or differences in the physical environment. Even for multifaceted interventions-with most studies and participants-the results are inconclusive, and it may be that for these interventions and for hip protectors there is insufficient power to draw definitive conclusions about effects on fracture rate, meaning that even these studies deserve further replication, perhaps after further work on improving targeting and adherence. Despite the pressing importance of preventing falls and fractures in hospitals and care homes, the evidence is inconclusive for multifaceted interventions in care homes and single interventions (except hip protectors) in either setting in reducing fall rates, risk of falling, or fracture rates. Use of hip protectors in care homes is associated with a small reduction in rates of hip fracture, which despite borderline significance on meta-analysis is inconclusive because of clustering effects. More encouragingly, we found a modest reduction in the rate of falls with a multifaceted intervention in hospital settings (though we found no effect on risk of falling or fracture rates). It therefore seems likely that currently healthcare providers are incurring important opportu- 
nity and financial costs by using injury prevention strategies of uncertain value.

We thank Karen Stenner (collation and summary of papers) Jill Duncan, (construction of literature search and retrieval of articles), and Jane Hardie (secretarial and administrative work).

Contributors: DO was principal investigator, was responsible for design, quality assessment, review of studies, and writing up, and is guarantor. MAG, CRV, JBC, FCM, and FES were also responsible for design, quality assessment, review of studies, and writing up. YG and AW were responsible for design, numerical data abstraction, statistical analysis, meta-analysis, and quality assessment for statistical components. AV was responsible for literature review and economic evaluation.

Funding: Department of Health Accidental Injury Prevention Programme. Competing interests: None declared.

Ethical approval: Not required.

1 National Ageing and Research Institute. An analysis of research on preventing falls and falls injury in older people: community, residential and hospital settings. Canberra: Australian Government Department of Health and Ageing, 2004.

2 Healey F, Oliver D. Preventing falls and injury in hospitals. The evidence for interventions. Health Care Risk Rep 2006:June; 12-7.

3 Dickson B, Woodward M. Accident prevention in the elderly in nursing homes. 2nd year report. Newcastle: Gateshead, South Tyneside, Sunderland and Northumberland Registration and Inspection Areas for Care Homes, 2000.

4 Rubenstein LZ, Josephson KR, Osterweil LD. Falls and fall prevention in the nursing home. Clin Geriatr Med 1996;12:881-902.

5 Mahoney J. Immobility and falls. Clin Geriatr Med 1998;14:699-79.

6 Morgan I, Sahota O, Wenn R, Moran C. Incidence of additional limb fractures in elderly patients presenting to secondary care with an osteoporotic hip fracture. Osteoporos Int 2004;supp 2:S30.

7 Vetter N, Ford D. Anxiety and depression scores in elderly fallers. Int J Geriatr Psych 1989;4:168-73

8 Murphy J, Isaacs B. The post fall syndrome. A study of 36 elderly inpatients. Gerontology 1982:28:265-70.

9 Bates D, Pruess K, Souney P, Platt R. Serious falls in hospitalized patients: correlates and resource utilization. Am J Med 1995;99:137-43.

10 Liddle J, Gilleard C. The emotional consequences of falls for patients and their families. Age Ageing 1994;23(supp 4): 17 .

11 Oliver D. Bed falls and bedrails. What should we do? Age Ageing 2002;31:415-8.

\section{What is already known on this topic}

Falls are the most common adverse incident in hospitals and care homes, nearly always affecting frail elderly people, many of whom have dementia or delirium

Risk management must be balanced against the need to promote functional independence and to respect autonomy

Previous reviews and guidelines have focused largely on elderly people living in the community and those without cognitive impairment

\section{What this study adds}

There is evidence for modest reductions in fall rates in hospital patients from multifactorial interventions and on hip fractures from hip protectors in care home residents

There is insufficient evidence for any other interventions in these settings
12 Gillespie LD, Gillespie WJ, Robertson MC, Lamb SE, Cumming RG, Rowe BH. Interventions for preventing falls in elderly people. Cochrane Database Syst Rev 2006;(4):CD0003402.

13 National Institute for Health and Clinical Excellence. Falls. The assessment and prevention of falls in older people. London: NICE, 2004.

14 Moher D, Cook D, Eastwood S, Olkin I, Rennie D, Stroup DF. Improving the quality of reports of meta-analyses of randomised controlled trials: the QUOROM statement. Quality of reporting of meta-analyses. Lancet 1999;354:1896-900.

15 Mulrow C, Oxman A. Cochrane reviewers'handbook [updated 1996] in Review Manager (RevMan) [computer programme] Version 3.1. Oxford: Cochrane Collaboration, 1997.

16 Downs SH, Black $\mathrm{N}$. The feasibility of creating a checklist for the assessment of the methodological quality both of randomised and non-randomised studies of health care interventions. J Epidemiol Community Health 1998;52:377-84.

17 Whitehead A. Meta-analysis of controlled clinical trials. Wiley: Chichester, 2002.

18 DerSimonian R, Laird N. Meta-analysis in clinical trials. Control Clin Trials 1986;7:17788.

19 Campbell M, Elbourne D, Altman D. Consort statement: extension to cluster randomised controlled trials. BMJ 2004;328:702-8.

20 Higgins JPT, Thompson SG. Quantifying heterogeneity in a meta-analysis. Stat Med 2002;21:1539-58

21 Thompson SG, Sharp SJ. Exploring heterogeneity in meta-analysis: a comparison of methods. Stat Med 1999;18:2693-708.

22 Simpson AH, Lamb S, Roberts PJ, Gardner TN, Evans JG. Does the type of flooring affect the risk of hip fracture? Age Ageing 2004;33:242-6.

23 Healey F. Does flooring type affect the risk of injury in older inpatients? Nurs Times 1994;90:40-1.

24 Flicker L, MacInnis RJ, Stein MS, Scherer SC, Mead KE, Nowson CA, et al. Should older people in residential care receive vitamin $\mathrm{D}$ to prevent falls? Results of a randomized trial. JAm Geriatr Soc 2005;53:1881-8.

25 Law M. Withers H, Morris J, Anderson F. Vitamin D supplementation and the prevention of fractures and falls: results of a randomised trial in elderly people in residential accommodation. Age Ageing 2006;35:482-6.

26 Ray WA, Taylor JA, Meador KG, Thapa PB, Brown AK, Kajihara HK, et al. A randomized trial of a consultation service to reduce falls in nursing homes. JAMA 1997:278:557-62.

27 Campbell M, Fitzpatrick R, Haines A, Kinmonth AL, Sandercock P, Spiegelhalter D, et al. Framework for design and evaluation of complex interventions to improve health. BMJ 2000;321:694-6.

28 Connelly J. Critical realism and health promotion: effective practice needs an effective theory. Health Educ Res 2001;16:115-9.

29 Herbert RD, Bo K. Analysis of quality of interventions in systematic reviews. $B M J$ 2005;331:507-509.

30 Oakley A, Strange V, Bonell C, Allen E, Stephenson J, Ripple Study Team. Process evaluation in randomised controlled trials of complex interventions. BMJ 2006;332:413-6.

(Accepted 20 October 2006)

doi $10.1136 /$ bmj.39049.706493.55

University of Reading Institute of Health Sciences, Reading RG1 5AG

David Oliver senior lecturer

James B Connelly professor

Christina R Victor professor

Margot A Gosney professor

Institute for Ageing and Health, University of Newcastle upon Tyne, Newcastle

NE4 6BE

Fiona E Shaw honorary clinical senior lecturer

University of Reading Medical and Pharmaceutical Statistics Research Unit, Reading RG6 6FN

Anne Whitehead professor

Department of Biostatistics, Faculty of Medicine, Ankara University, 06100 Ankara, Turkey

Yasemin Genc lecturer

Centre for Health Services Research, University of Newcastle upon Tyne,

Newcastle NE2 4AA

Alessandra Vanoli lecturer

Department of Ageing and Health, Guy's and St Thomas' NHS Foundation Trust, London SE1 7EH

Finbarr C Martin consultant geriatrician

Correspondence to: D Oliver d.oliver@reading.ac.uk 